\title{
1 Sn-inserted Al-induced layer exchange for large-grained GeSn thin films
}

on insulator

5 305-8573, Japan

6 E-mail: toko@bk.tsukuba.ac.jp

7

Large-grained polycrystalline GeSn layers on glass are achieved through the layer exchange between a-Ge and Sn-doped Al layers. The thicker Sn layers, inserted below Al layers, provided the faster growth velocity, resulting in the smaller grain size of the GeSn layer. Controlling the Sn thickness $(10 \mathrm{~nm})$ and the growth temperature $\left(300^{\circ} \mathrm{C}\right)$ allowed for approximately 80\% (111)-oriented GeSn layer with grains having an average size of $40 \mu \mathrm{m}$. The lower growth temperature led to the higher Sn content in $\mathrm{GeSn}: 300{ }^{\circ} \mathrm{C}$ resulted in a Sn content of $2 \%$. These findings are meaningful to researches related to GeSn on insulators for fabricating advanced electrical and optical devices on inexpensive substrates as well as on $\mathrm{Si}$ platforms.

Keywords: Metal-induced crystallization; Al-induced layer exchange; Crystal orientation; Solid phase crystallization; Polycrystalline films; Semiconducting germanium 


\section{Introduction}

GeSn offers exciting possibilities as a next-generation material for advanced electronic and photonic devices based on group-IV semiconductors [1,2]. Although the solubility limit of $\mathrm{Sn}$ in Ge is as low as $1 \%$, non-equilibrium low-temperature process using molecular-beam epitaxy (MBE) [3-6], chemical-vapor deposition (CVD) [7,8], or ion implantation [9] have allowed for Sn contents more than the solubility limit. These studies have demonstrated the advantage of GeSn: the carrier mobility of GeSn exceeded that of Ge $[10,11]$; the infrared detection capability, covering all the relevant telecommunications wavelengths, is extended by Sn contents over 2\% [12-14]; high substitutional Sn content (> $6 \%$ in GeSn provides the direct transition in the near infrared region, allowing for lasing $[15,16]$.

The study of GeSn on insulators (GSOIs) has been accelerated for fabricating monolithically integrated GeSn-based devices on three-dimensional Si large-scale integrated circuits and on multi-functional displays with glass or plastic substrates [17-26]. The incorporation of $2 \%$ Sn into Ge passivated the vacancy defects or reduced the grain boundary scattering, resulting in the higher carrier mobilities than Ge $[18,19]$. The tin-induced crystallization (TIC) of amorphous $\mathrm{Ge}(\mathrm{a}-\mathrm{Ge})$ has recently garnered attention [23-26]. We controlled the Sn content in the resulting polycrystalline (poly-) GeSn by tuning the TIC temperature and achieved high-Sn (25\%) content poly-GeSn on glass at $70{ }^{\circ} \mathrm{C}[26]$. The grain sizes of the poly-GeSn layers formed by TIC, however, were small $(<1 \mu \mathrm{m})$, which would deteriorate the electrical properties of the GeSn layer.

For fabricating a large-grained ( $>100 \mu \mathrm{m})$ poly-Ge on insulators, layer exchange growth between metal and a-Ge layers has been widely investigated using $\mathrm{Al}$ [27-31] or $\mathrm{Au}$ 
[32-33]. Besides, the layer exchange technique enables us to form (111)-oriented Ge [31-33], which is preferable for Ge devices [34,35]. In the preset paper, we studied a way using Sn-doped Al-induced crystallization (AIC) to form a large-grained, (111)-oriented poly-GeSn thin film on an insulator. The Sn-doping facilitated the growth and resulted in a 2\% Sn-content GeSn layer with $40 \mu \mathrm{m}$ grains.

\section{Experimental details}

Figure 1 shows the process of sample preparation. The Sn (thickness: 0-20 nm) and $\mathrm{Al}$ (thickness: $50 \mathrm{~nm}$ ) layers were prepared in sequence on $\mathrm{SiO}_{2}$ glass substrates. After the depositions, the $\mathrm{Al}$ layers were exposed to air for 10 min to form native $\mathrm{AlO}_{x}$ membranes as diffusion limiting layers, which significantly influence the growth properties of AIC. From our previous study, the thickness of the $\mathrm{AlO}_{x}$ membrane is estimated to be approximately 2 nm [29]. After that, 60-nm-thick undoped a-Ge layers were prepared. All depositions were carried out at room temperature using a radio-frequency magnetron sputtering method. The samples were then annealed at $300-350{ }^{\circ} \mathrm{C}$ for $100 \mathrm{~h}$ in $\mathrm{N}_{2}$ ambient. Here we expected the following reactions: the Sn and $\mathrm{Al}$ layers were mixed each other during annealing because the Sn-Al eutectic point is $231{ }^{\circ} \mathrm{C}$, and then the a-Ge layer is crystallized in GeSn via the layer exchange, as schematically shown in Fig. 1.

After the completion of the layer exchange, the Ge islands and Al layer, remaining in the top layer, are sequentially removed using $50 \% \mathrm{H}_{2} \mathrm{O}_{2}$ solution for $30 \mathrm{~min}$ and $1.5 \% \mathrm{HF}$ solution for $1 \mathrm{~min}$ [31]. The resulting layers were characterized by using Nomarski optical microscopy, a $\theta$-2 $\theta$ X-ray diffraction (XRD) measurement (spot size: $10 \mathrm{~mm}$ ), and electron backscatter diffraction (EBSD) analysis. 


\section{Results and discussion}

Figure 2 shows Nomarski optical micrographs of the back surfaces of the $300{ }^{\circ} \mathrm{C}$

annealed samples with and without a 10 -nm-thick Sn layer. As representatively shown in Fig. 2, at $300{ }^{\circ} \mathrm{C}$, the layer exchange of the samples with the thin Sn layers (0-5 nm) did not finish within $100 \mathrm{~h}$, while that of the samples with the thick Sn layers $(10-20 \mathrm{~nm})$ finished. These results mean that the growth velocity increased with increasing the initial Sn thickness. This behavior, i.e., the growth velocity enhancement of a-Ge using Sn as a catalyst, is consistent with the previous reports $[25,26]$. This is because the temperature of the reaction between Sn and a-Ge is quite low. On the other hand, at $350{ }^{\circ} \mathrm{C}$, the samples with the thick Sn layers (> $10 \mathrm{~nm})$ led to the crystallization of a-Ge without layer exchange, resulting in fine grains $(<1$ $\mu \mathrm{m})$ according to the EBSD measurement. This is likely owing to the diffusion of Sn into the a-Ge layer, which crystallized the a-Ge layer before layer exchange started. Note that the thickness of the resulting $\mathrm{Ge}(\mathrm{Sn})$ layer was the same as the total thickness of the initial $\mathrm{Al}$ and Sn layers. The surface roughness of the $\mathrm{Ge}(\mathrm{Sn})$ was measured using atomic force microscopy. The root mean square value was found to be $4.3 \mathrm{~nm}$, which was almost the same as that of the $\mathrm{Al}$ layer stacked on the Sn layer before annealing. These results suggest that the mixed Al-Sn layer acted as a catalyst for layer exchange growth of a-Ge [30].

Figure 3 shows the EBSD images of the samples grown via layer exchange, indicating that the crystal orientation of the resulting $\mathrm{Ge}(\mathrm{Sn})$ layers is significantly affected by the Sn insertion as well as the growth temperature. Figures 3(a) and 3(c) show that the samples without Sn layers are highly (111)-oriented, consistent with our prior studies using 
50-nm-thick Al layers [29,30]. When the Sn layers are inserted, the resulting Ge(Sn) layers have (100)-oriented regions. This phenomenon is pronounced for the samples with thicker Sn layers and higher growth temperatures, as shown in Figs. 3(b), 3(e), and 3(f). Thus, the AIC-Ge(Sn) layers are (111)- or (100)-oriented. This behavior is the same as AIC-Si, where the interfacial energy of Si nuclei in Al becomes minimum in (111) or (100) planes depending on the growth conditions [36]. By tuning the Sn thickness and growth temperatures, (111)-oriented Ge layers can be obtained, as shown in Figs. 3(d) and 3(g).

The crystal orientation fraction and the average grain size of the resulting $\mathrm{Ge}(\mathrm{Sn})$ layers were estimated from the EBSD images shown in Fig. 3. Figures 4(a)-4(c) respectively indicate that the thicker Sn layer provides the lower (111) orientation fraction, the higher (100) orientation, and the smaller grain size. This is a typical behavior when the growth velocity in AIC is promoted [37,38]: the insertion of the Sn layer likely facilitated the nucleation other than (111) orientation, and reduced the size of the resulting grains. Thus, by lowering the growth temperature, i.e., lowering the growth velocity, the (111) orientation and the grain size are improved. For all the samples grown via the layer exchange, the grain sizes are no less than $20 \mu \mathrm{m}$, which is large enough for device fabrications.

The Sn content in the resulting layers was evaluated by using a $\theta-2 \theta$ XRD measurement. Figure 5 (a) shows the XRD patterns of the samples annealed at $325{ }^{\circ} \mathrm{C}$. The peaks at around $27^{\circ}$, originated from the $\mathrm{Ge}(111)$ plane, are observed for all the samples. In the measured $2 \theta$ range $\left(20-60^{\circ}\right)$, other peaks were not observed because the poly-GeSn layers were thin and preferentially (111)-oriented. With increasing the thickness of the inserted Sn layer, the peak intensity is further weakened. This behavior is attributed to the lower (111) orientation fraction, as shown in Fig. 4(a). The detailed analysis clarified that 
115 the peak positions of the samples with a Sn layer slightly shifted to smaller angles,

116 suggesting the formation of GeSn containing substitutional Sn atoms [26]. The Sn contents

117 were calculated from the peak positions assuming the validity of Vegard's law. The results

118 are shown in Fig. 5(b). When the Sn layer is thin, the Sn content in GeSn increases as the

119 Sn thickness increases. On the other hand, when the Sn layer is thick, the Sn content in

$120 \mathrm{GeSn}$ is constant regardless of the Sn thickness. The saturated Sn content is determined by

121 the annealing temperature: the lower temperature provides the higher Sn content. The maximum Sn content of $2 \%$ was obtained at $300{ }^{\circ} \mathrm{C}$. We evaluated the electrical properties of the poly-GeSn, formed at $300{ }^{\circ} \mathrm{C}$ with a 10 -nm-thick Sn layer, using Hall measurement.

124 The carrier mobility was $20 \mathrm{~cm}^{2} / \mathrm{Vs}$ and the hole concentration was $3.5 \times 10^{20} \mathrm{~cm}^{-3}$. These

125

126 values are almost the same as those of the Al-doped Ge layer formed by conventional AIC. Figure 5 (c) shows that the Sn content clearly increases with decreasing the annealing temperatures and that the result of AIC-GeSn is consistent with that of TIC-GeSn [26] on the perspective of the relationship between the Sn content and growth temperature. These results suggest that the maximum $\mathrm{Sn}$ content in GeSn is limited by the growth temperature when the GeSn is formed as a result of the reaction between Sn and a-Ge. This is because that the saturated Sn concentration is likely determined by both the solubility limit of Sn in Ge (approximately 1\%) and the non-equilibrium fluctuation, depending on the process temperature. Lowering the growth temperature in AIC using growth promotion techniques, reported in Ref. 30, will yield a large-grained GeSn layer with a higher Sn content.

\section{Conclusions}

The Sn-doped AIC of a-Ge, initially inserting Sn layers below $\mathrm{Al}$, was 
138 investigated to obtain a large-grained GeSn layer on insulators. Thickening the Sn layer

139 facilitated the growth velocity of AIC, deteriorating the grain size and (111) orientation

140 fraction of the resulting GeSn layer. By tuning the thickness of the Sn layer $(10 \mathrm{~nm})$ and

141 the growth temperature $\left(300{ }^{\circ} \mathrm{C}\right)$, a large-grained $(\sim 40 \mu \mathrm{m}),(111)$-oriented $(\sim 80 \%) \mathrm{GeSn}$

142 layer on glass was achieved. Those growth properties are the advantages over the previous

143 poly-GeSn layers formed by the solid-phase crystallization of a-GeSn. The Sn content in the

144 GeSn layer was 2\%, which was determined by the growth temperature. These findings are

145 meaningful to the research on GSOIs for fabricating high-performance electrical and optical

146 devices on inexpensive substrates.

147

148 Acknowledgments

149 This work was financially supported by the JGC-S Scholarship Foundation and the Japan

150 Prize Foundation. Some experiments were conducted at the International Center for Young

151 Scientists in NIMS. 


\section{References}

153 [1] K. Alberi, J. Blacksberg, L. Bell, S. Nikzad, K. Yu, O. Dubon, W. Walukiewicz, Band anticrossing in 154 highly mismatched $\mathrm{Sn}_{x} \mathrm{Ge}_{1-x}$ semiconducting alloys, Phys. Rev. B 77 (2008) 073202.

155 [2] W.-J. Yin, X.-G. Gong, S.-H. Wei, Origin of the unusually large band-gap bowing and the breakdown of 156 the band-edge distribution rule in the $\mathrm{Sn}_{x} \mathrm{Ge}_{1-x}$ alloys, Phys. Rev. B 78 (2008) 161203.

157 [3] Y. Shimura, N. Tsutsui, O. Nakatsuka, A. Sakai, S. Zaima, Low temperature growth of $\mathrm{Ge}_{1-x} \mathrm{Sn}_{x}$ buffer 158 layers for tensile-strained Ge layers, Thin Solid Films 518 (2010) S2.

159 [4] M. Oehme, K. Kostecki, M. Schmid, M. Kaschel, M. Gollhofer, K. Ye, D. Widmann, R. Koerner, S. Bechler, E. Kasper, J. Schulze, Franz-Keldysh effect in GeSn pin photodetectors, Appl. Phys. Lett. 104 $161 \quad$ (2014) 161115.

[5] H. Lin, R. Chen, Y. Huo, T. I. Kamins, J. S. Harris, Raman study of strained $\mathrm{Ge}_{1-x} \mathrm{Sn}_{x}$ alloys, Appl. Phys. Lett. 98 (2011) 261917.

[6] A. A. Tonkikh, N. D. Zakharov, A. A. Suvorova, C. Eisenschmidt, J. Schilling, P. Werner, Cubic Phase Sn-Rich GeSn Nanocrystals in a Ge Matrix, Cryst. Growth Des. 14 (2014) 1617.

[7] V. R. D’Costa, J. Tolle, R. Roucka, C. D. Poweleit, J. Kouvetakis, J. Menéndez, Raman scattering in $\mathrm{Ge}_{1-y} \mathrm{Sn}_{y}$ alloys, Solid State Commun. 144 (2007) 240.

[8] B. Vincent, F. Gencarelli, H. Bender, C. Merckling, B. Douhard, D. H. Petersen, O. Hansen, H. H. (2011) 152103.

[9] K. Gao, S. Prucnal, R. Huebner, C. Baehtz, I. Skorupa, Y. Wang, W. Skorupa, M. Helm, S. Zhou, Ge $\mathrm{Ge}_{1-x} \mathrm{Sn}_{x}$ alloys synthesized by ion implantation and pulsed laser melting, Appl. Phys. Lett. 105 (2014) 042107.

[10] S. Gupta, Y. Huang, Y. Kim, E, Sanchez, K. C. Saraswat, Hole mobility enhancement in compressively strained $\mathrm{Ge}_{0.93} \mathrm{Sn}_{0.07}$ pMOSFETs, IEEE Electron Device Lett. 34 (2013) 831.

[11] R. R. Lieten, T. Maeda, W. Jevasuwan, H. Hattori, N. Uchida, S. Miura, M. Tanaka, J.-P. Locquet, Phase Epitaxy, Appl. Phys. Express 6 (2013) 101301.

[12] J. Mathews, R. Roucka, J. Xie, S. Q. Yu, J. Menendez, J. Kouvetakis, Extended performance 
182 [13] Y. Nakamura, N. Fujinoki, M. Ichikawa, Photoluminescence from Si-capped GeSn nanodots on Si 183 substrates formed using an ultrathin $\mathrm{SiO}_{2}$ film technique, J. Appl. Phys. 106 (2009) 014309.

184 [14] H. H. Tseng, H. Li, V. Mashanov, Y. J. Yang, H. H. Cheng, G. E. Chang, R. A. Soref, G. Sun, 185 GeSn-based p-i-n photodiodes with strained active layer on a Si wafer, Appl. Phys. Lett. 103 (2013) 231907.

186 [15] P. Moontragoon, R. A. Soref, Z. Ikonic, The direct and indirect bandgaps of unstrained $\mathrm{Si}_{x} \mathrm{Ge}_{1-x-y} \mathrm{Sn}_{y}$ and their photonic device applications, J. Appl. Phys. 112 (2012) 073106.

188 [16] S. Wirths, R. Geiger, N.V. Den Driesch, G. Mussler, T. Stoica, S. Mantl, Z. Ikonic, M. Luysberg, S. 189 Chiussi, J.M. Hartmann, H. Sigg, J. Faist, D. Buca, D. Grützmacher, Lasing in direct-bandgap GeSn alloy 190 grown on Si, Nat. Photonics 9 (2015) 88.

191 [17] O. Nakatsuka, K. Mochizuki, Y. Shimura, T. Yamaha, S. Zaima, Low temperature formation of $192 \mathrm{Si}_{1-x-y} \mathrm{Ge}_{x} \mathrm{Sn}_{y}$-on-insulator structures by using solid-phase mixing of $\mathrm{Ge}_{1-z} \mathrm{Sn}_{z} / \mathrm{Si}$-on-insulator substrates, Thin Solid Films 520 (2012) 3288.

194 [18] O. Nakatsuka, N. Tsutsui, Y. Shimura, S. Takeuchi, A. Sakai, S. Zaima, Mobility behavior of $\mathrm{Ge}_{1-x} \mathrm{Sn}_{x}$ layers grown on silicon-on-insulator substrates, Jpn. J. Appl. Phys. 49 (2010) 04DA10.

196 [19] W. Takeuchi, N. Taoka, M. Kurosawa, M. Sakashita, O. Nakatsuka, S. Zaima, High hole mobility tin-doped polycrystalline germanium layers formed on insulating substrates by low-temperature solid-phase crystallization, Appl. Phys. Lett. 107 (2015) 022103.

[20] M. Kurosawa, Y. Tojo, R. Matsumura, T. Sadoh, M. Miyao, Single-crystalline laterally graded GeSn on 200 insulator structures by segregation controlled rapid-melting growth, Appl. Phys. Lett. 101 (2012) 091905.

[21] M. Kurosawa, N. Taoka, H. Ikenoue, O. Nakatsuka, S. Zaima, Large grain growth of Ge-rich $\mathrm{Ge}_{1-\chi} \mathrm{Sn}_{x}$ $(\mathrm{x} \approx 0.02)$ on insulating surfaces using pulsed laser annealing in flowing water, Appl. Phys. Lett. 104 (2014) 203061901.

204 [22] H. Li, X. Wang, J. Liu, Pseudo single crystal, direct-band-gap $\mathrm{Ge}_{0.89} \mathrm{Sn}_{0.11}$ on amorphous dielectric layers 205 towards monolithic 3D photonic integration, Appl. Phys. Lett. 105 (2014) 201107.

206 [23] H. Li, J. Brouillet, A. Salas, X. Wang, J. Liu, Low temperature growth of high crystallinity GeSn on 207 amorphous layers for advanced optoelectronics, Opt. Mater. Express 3 (2013) 1385. 
[24] M. Kurosawa, N. Taoka, M. Sakashita, O. Nakatsuka, M. Miyao, S. Zaima, Liquid-Sn-driven lateral growth of poly-GeSn on insulator assisted by surface oxide layer, Appl. Phys. Lett. 103 (2013) 101904.

[25] H. Chikita, R. Matsumura, Y. Kai, T. Sadoh, M. Miyao, Ultra-high-speed lateral solid phase crystallization of GeSn on insulator combined with Sn-melting-induced seeding, Appl. Phys. Lett. 105 (2014) 202112.

[26] K. Toko, N. Oya, N. Saitoh, N. Yoshizawa, T. Suemasu, $70^{\circ} \mathrm{C}$ synthesis of high-Sn content (25\%) GeSn on insulator by Sn-induced crystallization of amorphous Ge, Appl. Phys. Lett. 106 (2015) 082109.

[27] Z.M. Wang, J.Y. Wang, L.P.H. Jeurgens, F. Phillipp, E.J. Mittemeijer, Origins of stress development during metal-induced crystallization and layer exchange: Annealing amorphous Ge/crystalline Al bilayers, Acta Mater. 56 (2008) 5047.

[28] S. Hu, A.F. Marshall, P.C. McIntyre, Interface-controlled layer exchange in metal-induced crystallization of germanium thin films, Appl. Phys. Lett. 97 (2010) 082104.

[29] K. Toko, M. Kurosawa, N. Saitoh, N. Yoshizawa, N. Usami, M. Miyao, T. Suemasu, Highly (111)-oriented Ge thin films on insulators formed by Al-induced crystallization, Appl. Phys. Lett. 101 (2012) 072106.

[30] K. Toko, R. Numata, N. Oya, N. Fukata, N. Usami, T. Suemasu, Low-temperature $\left(180^{\circ} \mathrm{C}\right)$ formation of large-grained Ge ( 111 ) thin film on insulator using accelerated metal-induced crystallization, Appl. Phys. Lett. 104 (2014) 022106.

[31] K. Toko, K. Nakazawa, N. Saitoh, N. Yoshizawa, T. Suemasu, Improved Surface Quality of the Metal-Induced Crystallized Ge Seed Layer and Its Influence on Subsequent Epitaxy, Cryst. Growth Des. 15 (2015) 1535.

[32] J.-H. Park, K. Kasahara, K. Hamaya, M. Miyao, T. Sadoh, High carrier mobility in orientation-controlled large-grain $(\geq 50 \mu \mathrm{m}) \quad$ Ge directly formed on flexible plastic by nucleation-controlled gold-induced-crystallization, Appl. Phys. Lett. 104 (2014) 252110.

[33] H. Higashi, K. Kasahara, K. Kudo, H. Okamoto, K. Moto, J.-H. Park, S. Yamada, T. Kanashima, M. Miyao, I. Tsunoda, K. Hamaya, A pseudo-single-crystalline germanium film for flexible electronics, Appl. Phys. Lett. 106 (2015) 041902.

[34] T. Sasada, Y. Nakakita, M. Takenaka, S. Takagi, Surface orientation dependence of interface properties of 
$236 \mathrm{GeO}_{2} / \mathrm{Ge}$ metal-oxide-semiconductor structures fabricated by thermal oxidation, J. Appl. Phys. 106 (2009)

$237 \quad 073716$.

238 [35] T. Nishimura, C. H. Lee, T. Tabata, S. K. Wang, K. Nagashio, K. Kita, A. Toriumi, 239 High-Electron-Mobility Ge n-Channel Metal-Oxide-Semiconductor Field-Effect Transistors with 240 High-Pressure Oxidized $\mathrm{Y}_{2} \mathrm{O}_{3}$, Appl. Phys. Express 4 (2011) 064201.

241 [36] A. Sarikov, J. Schneider, J. Berghold, M. Muske, I. Sieber, S. Gall, et al., A kinetic simulation study of 242 the mechanisms of aluminum induced layer exchange process, J. Appl. Phys. 107 (2010) 114318.

243 [37] K. Toko, R. Numata, N. Saitoh, N. Yoshizawa, N. Usami, T. Suemasu, Selective formation of 244 large-grained, (100)- or (111)-oriented Si on glass by Al-induced layer exchange, J. Appl. Phys. 115 (2014) $245 \quad 094301$.

246 [38] M. Kurosawa, T. Sadoh, M. Miyao, Comprehensive study of Al-induced layer-exchange growth for 247 orientation-controlled Si crystals on $\mathrm{SiO}_{2}$ substrates, J. Appl. Phys. 116 (2014) 173510. 


\section{$250 \quad$ Figure Captions}

Fig. 1. Schematic of the sample preparation.

253

Fig. 2. Nomarski optical micrographs of the back surfaces of the $300{ }^{\circ} \mathrm{C}$ annealed samples (a) without and (b) with a 10-nm-thick Sn layer.

Fig. 3. EBSD images of the $\mathrm{Ge}(\mathrm{Sn})$ layers in the normal direction, summarized as a matrix of the initial Sn thickness $(0,5,10$, and $20 \mathrm{~nm})$ and the annealing temperature (300, 325, and $350{ }^{\circ} \mathrm{C}$ ). The coloration indicates crystal orientation, as shown in the legend.

Fig. 4. Initial Sn thickness dependence of (a) (111) orientation fraction, (b) (100) orientation fraction, and (c) average grain size in the AIC-GeSn layers annealed at $300{ }^{\circ} \mathrm{C}$ (circles), $325^{\circ} \mathrm{C}$ (squares), and $350{ }^{\circ} \mathrm{C}$ (triangles) for $100 \mathrm{~h}$.

Fig. 5. (a) $\theta-2 \theta$ XRD patterns of the AIC-GeSn layers with initial Sn thickness of $0-20 \mathrm{~nm}$ after annealed at $325^{\circ} \mathrm{C}$ for 100 h. (b) Initial Sn thickness dependence of Sn concentration in AIC-GeSn after annealed at $300{ }^{\circ} \mathrm{C}$ (circles), $325{ }^{\circ} \mathrm{C}$ (squares), and $350{ }^{\circ} \mathrm{C}$ (triangles) for $100 \mathrm{~h}$, derived from the XRD peak position of Ge(111) using Vegard's law. (c) Annealing temperature dependence of Sn concentration in AIC-GeSn (open circles) and TIC-GeSn (open circles) from Ref. 26. 


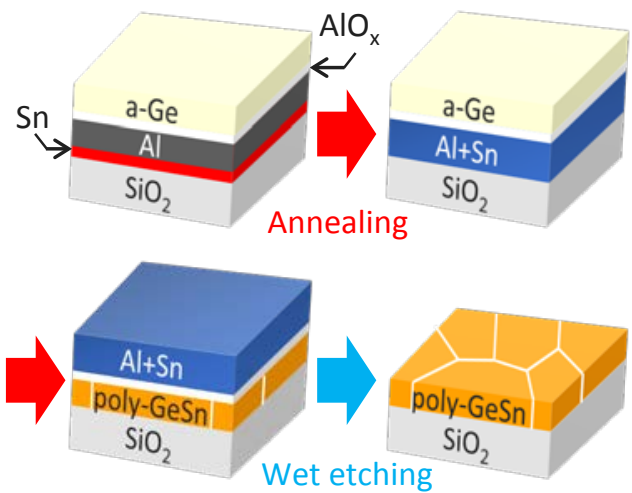

Figure 1 

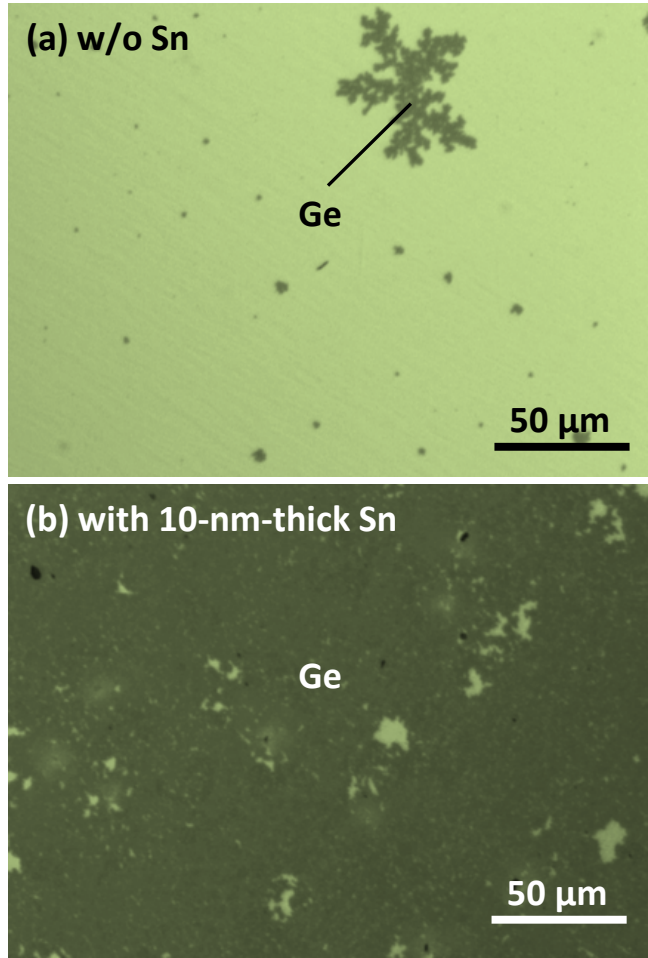

Figure 2 


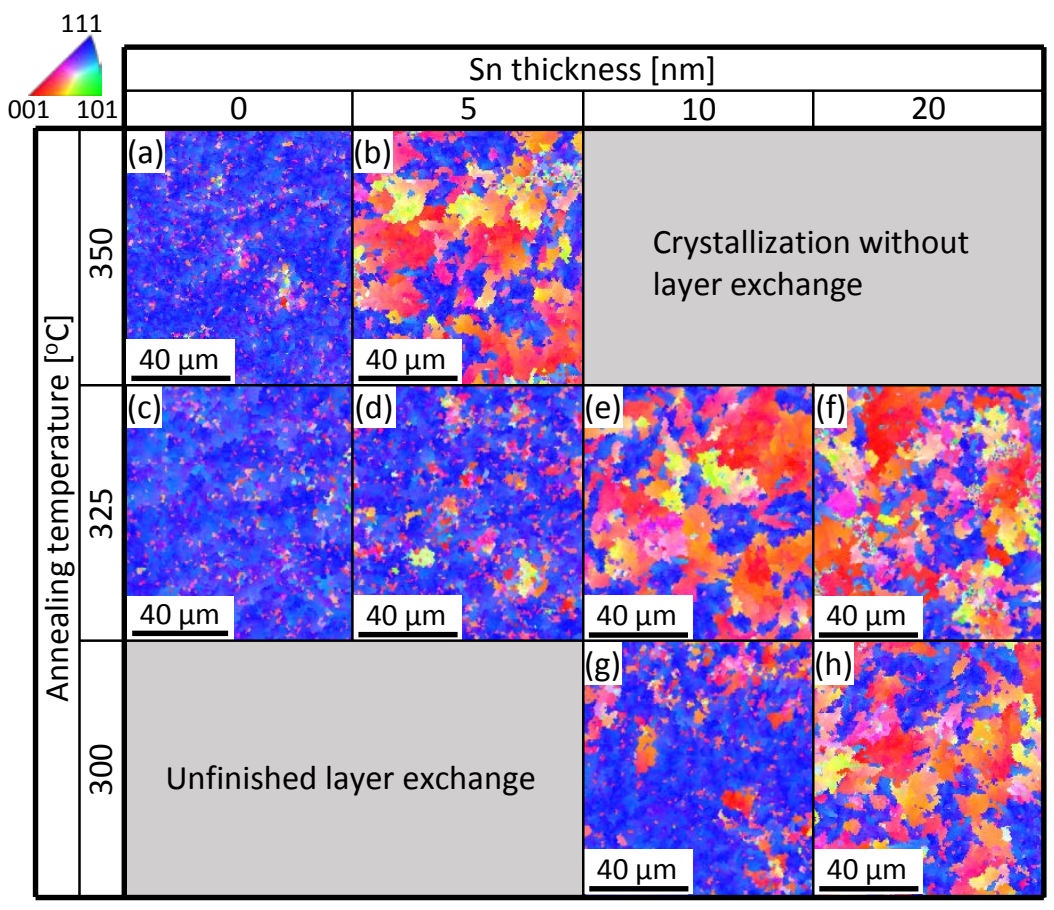

Figure 3 

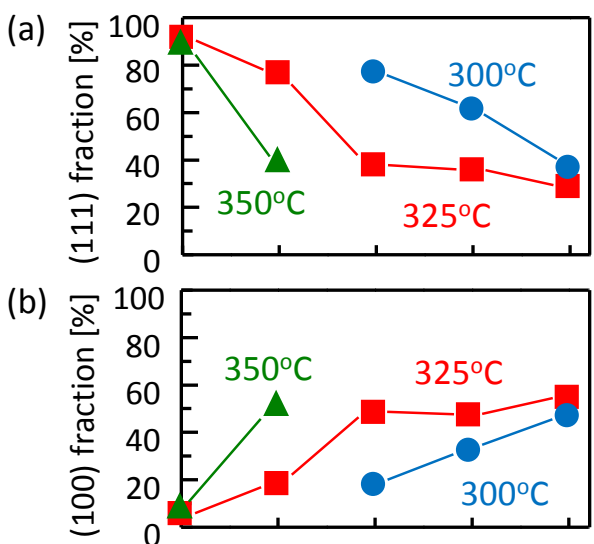

(c)

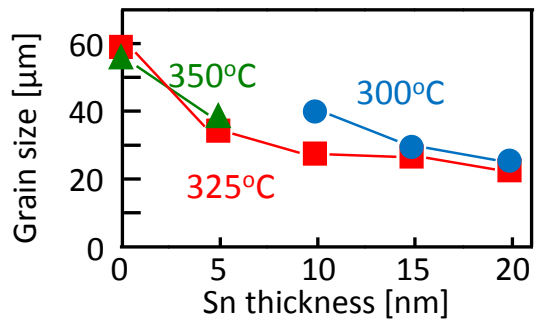

Figure 4 


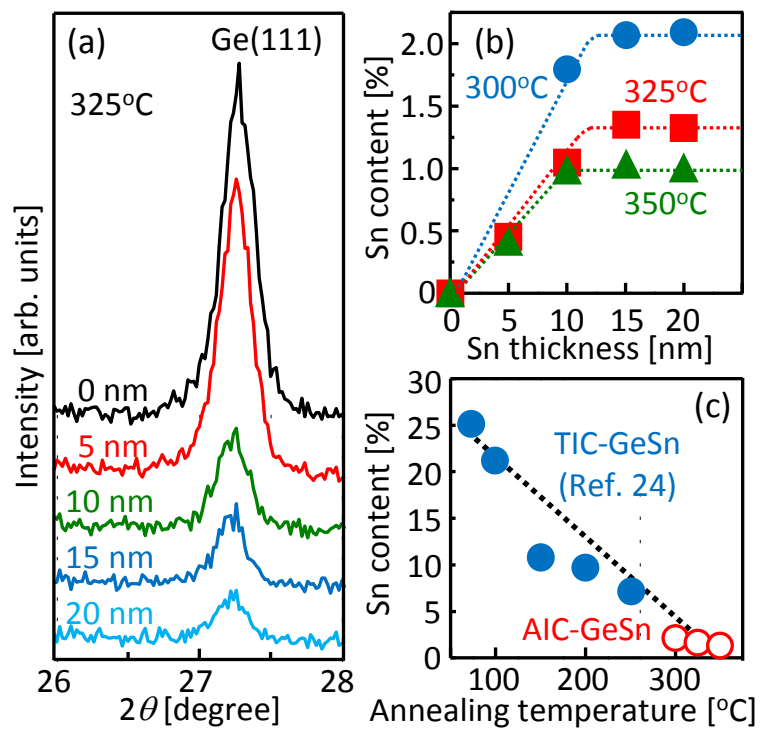

Figure 5 